\title{
Influenza Control in the Armed Forces
}

\author{
HERSCHEL E. GRIFFIN, M.D.
}

$\mathrm{T}$ THE HISTORY of the Armed Forces' interest in influenza is long and generally well known. Certainly the World War I experience has been recalled often enough recently to indicate the concern at least that far back. I)uring that period 1 in every 5 men in the Army contracted influenza, and roughly 25,000 died from its complications. This is nearly half the number of battle deaths in the American Expeditionary Forces. 'The disease is still potentially important to the military as well as to the population at large.

The modern history of the Armed Forces' activities directed toward the control of influenza dates from December 27,1940 . On that date It. Col. James Stevens Simmons, the late dean of the Harvard School of Public Health, wrote a letter recommending the establishment of a "Board for the Investigation and Control of Influenza and Other Epidemic I)iseases in the Army." Within 15 days approval was granted by the War Department, and the board, with its formidable title, was established. This agency, now the Armed Forces Epidemiological Board, and its Commission on Influenza are still active. The continuity of the agency is apparent from the fact that two members of the early commission are now on the board, one as its president, and two others are now directors of other commissions.

The major influenza control activities of the

Lieutenant Colonel Griffin, MC, U.S. Army, is chief of the Communicable Disease Branch, Preventive Medicine Division, Office of the Surgeon General, Department of the Army. This paper is based on comments at a symposium on Asian influenza held by the American College of Preventive Medicine and the health officers' section at the 1957 meeting of the American Public Health Association. three military departments could be classified in several different ways. I have chosen to consider them under four main headings: surveillance, investigations, vaccination, and medical care. I shall briefly describe the activities of each of these areas and report our experience so far.

\section{Military Surveillance}

Surveillance is maintained, as in civilian public health, by periodic reporting augmented by special epidemiological reports when the occasion warrants. For example, the Army has 176 stations around the world which submit weekly telegraphic reports enumerating new cases diagnosed. Common respiratory disease, influenza, and pneumonia are among the reportable categories. By this means trends may be determined and the overall picture seen. More detailed information is submitted by special telegraphic reports in outbreaks of respiratory disease.

Laboratory surveillance is based on the central laboratory at the Walter Reed Army Institute of Research and extends through the area laboratories in this country and overseas. Each has facilities for viral isolation and serologic identification.

Military medical facilities also participate in civilian reporting programs. Station medical officers comply with the State and local reporting requirements. In turn the three Surgeons General report their experience to the Public Health Service for publication in the Morbidity and Mortality Weekly Report. Coordination of effort among military, State, Public Health Service, and World Health Organization laboratories has been close from the beginning of man's ability to type the virus. 
Through this surveillance an increasingly clear picture of Asian influenza among the military has emerged.

The most important characteristic of influenza to the military in 1957 was its high attack rate. This was the predominant feature of the early epidemics in Asian populations. But when the epidemic reached our military and naval units in the Far East, where we could focus closer investigative study, it became apparent that the attack rate was not constant. In some units the rate reached 65 percent, in others 10 to 20 percent, and occasionally there were only a few cases. This variation, it was soon determined, was not due to preexisting immunity since antibodies to this virus were entirely lacking in this group. It appeared to be a function of crowding and closeness of contact.

The high rates occurred in units where the men lived, slept, ate, and worked together, such as those aboard naval vessels and in recruit training camps. When the populations were not so concentrated, the rates were low. For example, the outbreak at the San Diego Naval Training Center began so explosively that many first thought it would turn out to be adenovirus disease. Moreover, the rates in recruits were high, and in the cadre, who lived in their own homes, the rates were low. But it was influenza. This pattern has now been repeated many times.

A companion feature has been rapidity of spread, or perhaps more precisely the almost simultaneous occurrence of geographically widely separated outbreaks. How the disease first swept through Asia is well known. Its successive occurrence in countries around the world and the early widespread seeding in the United States have been described.

The first confirmed military outbreak in this country occurred in an east coast naval station early in June 1957. For 3 months the laboratories across the country reported occasional virus isolations and significant titer rises from sporadic cases. But there were few outbreaks. Although the virus was widespread, the respiratory disease rate for Army troops in the United States remained relatively constant, below 100 per 1,000 per year in the weekly reports.
Then, in the first week of September, many special epidemiological reports were received. The rate for Army personnel in the United States rose in 5 weeks to more than 750 cases per 1,000 per year, as some 30,000 cases occurred. By mid-October, though, the rate was headed precipitiously downward again. At the end of the first week in December it was down to 215. Thus, although person-to-person spread may account for infection with the virus, it alone cannot explain the pattern of the occurrence of epidemic influenza in the Army. The pattern in naval fleet units is a little clearer because of the closeness of the contact and more nearly resembles the spread in Asia or the occurrence of disease in families.

If one focuses attention upon individual bases and ships rather than upon the armed services as a whole, an important mitigating feature of the disease is brought to light. This is the short duration of outbreaks. In military experience most have run their course in 4 to 6 weeks, except where there have been additions to the population. This brevity of duration significantly lessens the effect of even intense outbreaks. In those outbreaks where the overall attack rate reached 25 percent, only 5 to 6 percent were ill at the peak, and then for only a few days. Prevalence is a better index of impact of disease than is incidence.

The laboratory has contributed significantly to surveillance. Its first function is categorization of the outbreak through confirmation of the diagnosis. Our practice is not to submit every case of respiratory disease to the laboratory for study, but to investigate throat washings and serum pairs from a small sample of characteristic cases early in the outbreak. Once the outbreak is categorized as influenza, cases are diagnosed clinically.

Early in the summer it became apparent that "all is not flu that fevers." Outbreaks presumptively diagnosed as influenza were proved in the laboratory to be as diverse as streptococcal exudative pharyngitis, common cold, and salmonellosis. One wonders at the reliability of the cumulative numbers being generally quoted.

The laboratories have been examining the homogeneity of the virus. Remember that early in 1957 an influenza $A^{\prime}$ virus was widely 
disseminated. In April the Asian variant started its world tour. The question has been raised as to whether another strikingly different $A$ virus would appear this year. Experience would say no, and so far the laboratory has borne out experience. But vigilance is being maintained for the early detection of the unexpected, should it occur.

Surveillance, then, has identified the close, continuous contact, such as that of the family or the crew of a ship, as the hazardous contact. Potential control measures involve preventing transmission of the virus within these close groups, rather than curtailing public gatherings. However, this does not preclude closing schools for administrative reasons because of high absentee rates or illness among teachers.

\section{Investigative Efforts}

Investigations are being carried out on many fronts so that when the time comes to draw conclusions about this epidemic, those conclusions may be based on data and not on speculation. We believe that we would be seriously remiss to allow this, the most significant occurrence of influenza in almost 40 years, to pass poorly or inadequately documented.

These investigations are being conducted at all levels. Prototype protocols for the epidemiological description of outbreaks at individual installations have been widely distributed. When they are returned, there will be a large body of information reported in comparable format, with data commonly categorized. We hope that this procedure will allow pooling of the data and permit an orderly and logical progression from the specific to the general when evaluations are made.

Military laboratories and research groups are also conducting studies. The early work of Dr. Maurice R. Hilleman and the Walter Reed Army Institute of Research group in identifying and categorizing the virus and in evaluating its antigenicity may be mentioned. Important work in clinical, laboratory, nursing, and other aspects of the situation is being done by other laboratories. We are continuing to finance the projects of the Armed Forces Epidemiological Board and its Commission on Influenza.
All this research, which represents a rather considerable commitment of personnel, facilities, and money, is not considered an ancillary function or an esoteric exercise, but an essential control activity. Just as the knowledge gained from the more than 15 years of previous study allowed prompt action when the new strains appeared, so the research carried out this year will continue to improve our position of preparedness for the future. At any rate, an effort is being made to avoid what Dr. Edward G. McGavran has categorized as the "loss of invaluable information through the failure to observe and record experience."

\section{Vaccination by the Services}

Vaccination represents the only really effective control measure available in combating influenza. The actual program of immunization is simple. Military personnel worldwide have received two doses of vaccine. Overseas, the same course was offered on a voluntary basis to civilian employees and dependent personnel. At installations in this country where supplies exceeded military need, one dose has been offered to dependents and civilian employees.

I have been asked to comment on the intracutaneous route of administering influenza vaccine.

The primary reason this route has been considered at all is that it enables the available supply of vaccine to reach a larger segment of the population. Hilleman and others have reported that the antibody response to intracutaneous vaccination was "almost as good" as the greater amount given subcutaneously. He noted that "this finding may be significant in situations where shortage of vaccine necessitates stretching the supply." Similarly, the special Committee on Influenza of the American Medical Association stated that the subcutaneous route was preferable, though the intracutaneous route was offered as a possible alternative.

In the Armed Forces all past studies and experience regarding the preventive effect of the vaccine, as opposed to antibody stimulation, have been based on the subcutaneous route. So this route was decided upon if the supply should prove sufficient. In one service, the supply for 
the first dose was limited and the intracutaneous route was used in part of its personnel. The subcutaneous route was used throughout for the second dose.

As yet we have no data on the protective effect of the intracutaneous injections. Studies are under way to provide factual data on which to base future action.

\section{Experiences in Medical Care}

It is not customary to consider medical care a control activity. But in this disease care and control are interrelated. Clinical features have epidemiological significance, and epidemiology guides care. There has been nothing peculiar to the military in its hospitalization and therapy policies. Conservatism has been the rule. Segregation of patients, preferably without hospitalization for the uncomplicated cases, and symptomatic therapy with avoidance of antibiotics have been practiced in military as well as in civilian medicine.

The first action taken in medical care was obviously operational planning. It was necessary to estimate the capability of the military medical services in order to provide personnel, supplies, and facilities to meet the load. Because these medical services are designed for rapid expansion of their reserve capacity, and personnel and supplies are mobile, it was concluded that the capability was sufficient to meet the anticipated load.

The first test of this conclusion was in the Philippines. Clark Air Force Base reported a sharp, severe outbreak, but needed no outside help to manage it. Now that there have been scores of such experiences, it has become clear that the military medical services are indeed capable of handling outbreaks of this magnitude. Even aboard ship, where expansion capability is limited, facilities have been adequate, though at times severely strained.

Clinical experience may be briefly summarized. Cases have been acute, with temperatures averaging about $103^{\circ} \mathrm{F}$. but recovery has been rapid. The average time lost has been about 4 days. Pneumonia diagnosed on clinical grounds has been seen in less than 1 percent of respiratory disease admissions. This includes primary pneumonia, with no association with influenza. In about $\check{5}$ percent of the respiratory cases where chest $\mathrm{X}$-rays have been taken routinely, some degree of pneumonitis has been reported.

Association of the influenza virus and a bacterial hitchhiker has not been observed, even in areas where streptococcal disease is endemic. In the Army one death has been associated with influenza. The few other pneumonia deaths have been intensively studied, but no evidence has been elicited which would indicate that influenza played a role in them.

In regard to the future, I can say only that this is our program; so far it has served us well. We intend to continue it as described, though ever alert to a change in the situation which would warrant modification or revision. We are prepared for such a change, while not necessarily predicting it. 